\title{
Assessing Best Practices for Grouping and Analyzing Urgent Care Center (UCC) and Emergency Department (ED) Data Sources within Syndromic Surveillance Systems
}

\author{
Melinda C. Thomas*, David Atrubin and Janet Hamilton \\ Bureau of Epidemiology, Florida Department of Health, Tallahassee, FL, USA
}

Objective

To identify best practices for grouping emergency department and urgent care data in a syndromic surveillance system.

\section{Introduction}

The Florida Department of Health (FDOH) electronically receives both urgent care center (UCC) data and hospital emergency department (ED) data from health care facilities in 43 of its 67 counties through its Electronic Surveillance System for the Early Notification of Community-based Epidemics (ESSENCE-FL). Each submitted record is assigned to one of eleven ESSENCE Syndrome categories based on parsing of chief complaint data. The UCC data come from 22 urgent care centers located in Central Florida, and the ED data come from 161 hospitals located across the state. Traditionally, the data from these two sources are grouped and viewed together. To date, limited investigation has been carried out on the validity of grouping data from UCCS and EDs in ESSENCE-FL. This project will investigate and describe the differences between the data received from these two sources and provide best practices for grouping and analyzing these data sources.

\section{Methods}

The ESSENCE-FL system was queried using "ER Data by Hospital Location by Hospital" with a weekly time resolution for facilities in the Central Florida region. The ESSENCE Syndrome Influenza-like Illness (ILI) was investigated during the period of Week 34, 2012 to Week 34, 2013. Chief complaint and discharge diagnosis data were analyzed for each facility type to investigate potential disparities in the data between the two sources. Findings based on all eleven ESSENCE Syndrome categories and the Other category will be presented. Data from all UCCs and EDs in the Central Florida region will be presented at the conference. A comparison of ESSENCE-FL system generated warnings and alerts from the data sets will also be discussed.

\section{Results}

ILI syndrome data from one UCC and one ED in geographic proximity in Central Florida were compared during Week 34, 2012 through Week 34, 2013 indicating that differences in the data would be most attributable to the type of facility. Of the 644 reported ILI syndrome visits at the UCC, $55.4 \%(\mathrm{n}=357)$ of the chief complaints contained the word "flu" or "flu like symptoms," $46.7 \%(n=301)$ "fever," 29.2\% (n=188) "cough," and 20.7\% (n=133) "sore throat." The ED had 1190 reported ILI syndrome visits with $24.3 \%$ (n=289) of the chief complaints contained the word "flu" or "flu like symptoms," $76.0 \%(\mathrm{n}=904)$ "fever," 54.7\% ( $\mathrm{n}=651)$ "cough," and 14.5\% ( $\mathrm{n}=173)$ "sore throat." $24.7 \%(\mathrm{n}=159)$ of the reported ILI syndrome visits at the UCC had a discharge diagnosis of Flu compared to $9.4 \%(\mathrm{n}=112)$ of those at the ED. Complaints falling into the Other syndrome category made up $60.1 \%$ of visits to the UCC but only $26.9 \%$ of visits to the ED.

\section{Conclusions}

Preliminary analysis indicates that earlier system generated alerts for flu are seen with UCC data compared to those based on ED data. Patients with a chief complaint of flu or flu like illness make up a larger percentage of ILI syndrome visits in the UCC than in the ED. The chief complaints falling into the ILI syndrome category were more likely to be diagnosed with flu at the UCC than at the ED. UCC data had a significantly larger percentage of chief complaints placed into the Other category. These results indicate that, while the data sources have often been grouped together for analysis purposes, there can be important differences that are lost in this practice. Utilizing UCC data could be a more timely method for the early warning detection of influenza activity.

\section{Keywords}

Syndromic surveillance; Public health informatics; Urgent care center; Emergency department

\section{Acknowledgments}

This project was supported in part by an appointment to the Applied Public Health Informatics Fellowship Program administered by CSTE and funded by the Centers for Disease Control and Prevention (CDC) Cooperative Agreement 3U38HM000414-04W1.

\section{*Melinda C. Thomas}

E-mail: melinda.thomas2@flhealth.gov 